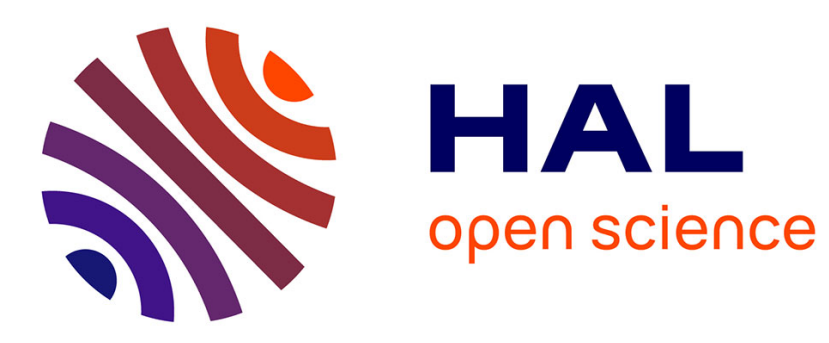

\title{
Quasi type IV codes over a non-unital ring
}

Adel Alahmadi, Alaa Altassan, Widyan Basaffar, Alexis Bonnecaze, Hatoon Shoaib, Patrick Solé

\section{To cite this version:}

Adel Alahmadi, Alaa Altassan, Widyan Basaffar, Alexis Bonnecaze, Hatoon Shoaib, et al.. Quasi type IV codes over a non-unital ring. Applicable Algebra in Engineering, Communication and Computing, 2021, 10.1007/s00200-021-00488-6 . hal-03173734

\section{HAL Id: hal-03173734 https://hal.science/hal-03173734}

Submitted on 18 Mar 2021

HAL is a multi-disciplinary open access archive for the deposit and dissemination of scientific research documents, whether they are published or not. The documents may come from teaching and research institutions in France or abroad, or from public or private research centers.
L'archive ouverte pluridisciplinaire HAL, est destinée au dépôt et à la diffusion de documents scientifiques de niveau recherche, publiés ou non, émanant des établissements d'enseignement et de recherche français ou étrangers, des laboratoires publics ou privés. 


\title{
Quasi type IV codes over a non-unital ring
}

\author{
Adel Alahmadi ${ }^{1}$ Alaa Altassan ${ }^{1} \cdot$ Widyan Basaffar $^{1} \cdot$ Alexis Bonnecaze $^{2}$. \\ Hatoon Shoaib ${ }^{1}$. Patrick Solé ${ }^{2}$
}

Received: 28 October 2019 / Accepted: 4 January 2021

(c) The Author(s), under exclusive licence to Springer-Verlag GmbH, DE part of Springer Nature 2021

\section{Abstract}

There is a local ring $I$ of order 4, without identity for the multiplication, defined by generators and relations as

$$
I=\left\langle a, b \mid 2 a=2 b=0, a^{2}=b, a b=0\right\rangle .
$$

We give a natural map between linear codes over $I$ and additive codes over $\mathbb{F}_{4}$, that allows for efficient computations. We study the algebraic structure of linear codes over this non-unital local ring, their generator and parity-check matrices. A canonical form for these matrices is given in the case of so-called nice codes. By analogy with $\mathbb{Z}_{4}$-codes, we define residue and torsion codes attached to a linear $I$-code. We introduce the notion of quasi self-dual codes (QSD) over $I$, and Type IV $I$-codes, that is, QSD codes all codewords of which have even Hamming weight. This is the natural analogue of Type IV codes over the field $\mathbb{F}_{4}$. Further, we define quasi Type IV codes over $I$ as those QSD codes with an even torsion code. We give a mass formula for QSD codes, and another for quasi Type IV codes, and classify both types of codes, up to coordinate permutation equivalence, in short lengths.

Widyan Basaffar

whbasaffar@kau.edu.sa

Adel Alahmadi

adelnife2@yahoo.com

Alaa Altassan

aaltassan@kau.edu.sa

Alexis Bonnecaze

Alexis.Bonnecaze@univ-amu.fr

Hatoon Shoaib

hashoaib@kau.edu.sa

Patrick Solé

sole@enst.fr

1 Math Dept, King Abdulaziz University, Jeddah, Saudi Arabia

2 CNRS, Centrale Marseille, I2M, Aix Marseille Univ, Marseille, France 
Keywords Rings $\cdot$ Codes $\cdot$ Additive $\mathbb{F}_{4}$-codes $\cdot$ Mass formulas $\cdot$ Type IV codes

Mathematics Subject Classification Primary 94 B05 · Secondary 16 A10

\section{Introduction}

While rings have been used as alphabets in Coding Theory for more than thirty years [14], it is only recently that non-unital rings have been used to that effect [1]. In that paper, the authors introduce the notion of Type IV codes over the ring $E$ in the terminology of $[8,13]$, in analogy with Type IV codes over unital rings of order four [7].

In this paper we study codes over another non-unital commutative ring of order four, denoted by $I$ in the classification of $[8,13]$. We call $I$-code of length $n$ any $I$-submodule of $I^{n}$. Such a code can be thought of as an additive $\mathbb{F}_{4}$-code in the sense of [3] with an extra module structure. This allows us to use the additive code package of Magma [12] in computations, and might lead in the future to the discovery of new additive $\mathbb{F}_{4}$-codes. In the ring $I$ the notion of self-dual code must be adapted since the usual relation between the size of the code and that of its dual [15], does not hold in general. We thus introduce, in Section 2.4, following [1], the notion of quasi self-dual code (QSD), that is of an I-code of length $n$ which is both selforthogonal and of size $2^{n}$. As with other local rings of order 4 , residue and torsion codes play a fundamental role in the structure of QSD codes. Thus, in Sect. 3, we derive a canonical form for the generator matrix of any $I$-code, based on the residue and torsion codes. However, an I-code cannot be determined uniquely by its residue and torsion codes. But it is completely characterized by the triplet formed by these two codes and a map between the residue code and the cosets of the torsion code. This is similar to the technique used to derive mass formulas for self-dual codes over rings [2, 5, 9]. In Sect. 4 we give a construction of QSD codes from a pair of binary codes; we call this the multilevel construction in analogy with a similar construction over $\mathbb{Z}_{4}$ [4]. In Sect. 5, we derive a mass formula for QSD codes with the objective of classifying these codes. The method of calculation of this formula leads us to introduce the notion of quasi Type IV codes: QSD codes whose torsion code is even, and to derive a mass formula for them. Finally, we give a classification of QSD codes up to length 3 in Sect. 6, and some concluding remarks in Sect. 7.

\section{Background material}

\subsection{Binary codes}

Denote by $w t(x)$ the (Hamming) weight of $x \in \mathbb{F}_{2}^{n}$, or, in other words, the number of indices $i$ where $x_{i} \neq 0$. A linear code is a subspace of $\mathbb{F}_{2}^{n}$, viewed as an $\mathbb{F}_{2}$-vector space. The dual of a binary linear code $C$ is denoted by $C^{\perp}$ and defined as 


$$
C^{\perp}=\left\{y \in \mathbb{F}_{2}^{n} \mid \forall x \in C,(x, y)=0\right\},
$$

where $(x, y)=\sum_{i=1}^{n} x_{i} y_{i}$, denotes the standard inner product. A code $C$ is selforthogonal if it is included in its dual: $C \subseteq C^{\perp}$. A code is even if all its codewords have even weight. All self-orthogonal codes are even but not conversely. Two binary codes are equivalent if there is a permutation of coordinates that maps one to the other.

\subsection{Rings}

Following [8], we define a ring on two generators $a, b$ by its relations

$$
I=\left\langle a, b \mid 2 a=2 b=0, a^{2}=b, a b=0\right\rangle .
$$

Thus, $I$ has characteristic two, and consists of four elements $I=\{0, a, b, c\}$, with $c=a+b$. The addition table is immediate from these definitions

$$
\begin{array}{|c|c|c|c|c|}
\hline+ & 0 & a & b & c \\
\hline 0 & 0 & a & b & c \\
\hline a & a & 0 & c & b \\
\hline b & b & c & 0 & a \\
\hline c & c & b & a & 0 \\
\hline
\end{array}
$$

The multiplication table is as follows.

$$
\begin{array}{|l|l|l|l|l|}
\hline \times & 0 & a & b & c \\
\hline 0 & 0 & 0 & 0 & 0 \\
\hline a & 0 & b & 0 & b \\
\hline b & 0 & 0 & 0 & 0 \\
\hline c & 0 & b & 0 & b \\
\hline
\end{array}
$$

From this table, we infer that this ring is commutative, and without an identity element for the multiplication. It is local with maximal ideal $J=\{0, b\}$, and residue field $\mathbb{F}_{2}=\{0,1\}$, the finite field of order 2 . Thus we have a $b$-adic decomposition as follows. Every element $i \in I$ can be written

$$
i=a s+b t,
$$

where $s, t \in \mathbb{F}_{2}$ and where we have defined a natural action of $\mathbb{F}_{2}$ on $I$ by the rule $r 0=0 r=0$ and $r 1=1 r=r$ for all $r \in I$. Thus $a=1 a, b=1 b$ and $c=a 1+b 1=c 1$. Note that for all $r \in I$, this action is "distributive" in the sense that $r(s \oplus t)=r s+r t$, where $\oplus$ denotes the addition in $\mathbb{F}_{2}$. On occasion we will use the inner product notation $(x, r)$ for $x \in \mathbb{F}_{2}^{n}, r \in I^{n}$ to denote

$$
(x, r)=\sum_{i=1}^{n} x_{i} r_{i}=\sum_{x_{i}=1} r_{i} .
$$


Denote by $\alpha: I \rightarrow I / J \simeq \mathbb{F}_{2}$ the map of reduction modulo $J$. Thus $\alpha(0)=\alpha(b)=0$, and $\alpha(a)=\alpha(c)=1$. This map is extended in the natural way in a map from $I^{n}$ to $\mathbb{F}_{2}^{n}$.

\subsection{Modules}

A linear $I$-code $C$ of length $n$ is an $I$-submodule of $I^{n}$. It can be described as the $I$-span of the rows of a generator matrix. An $\mathbb{F}_{4}$-code of length $n$ is just any non-empty subset of $\mathbb{F}_{4}^{n}$. The notion and notation of Hamming weight over $\mathbb{F}_{4}$ is the same as over $\mathbb{F}_{2}$. An additive code of length $n$ over $\mathbb{F}_{4}$ is an additive subgroup of $\mathbb{F}_{4}^{n}$. It is an $\mathbb{F}_{2}$ vector space with $4^{k}$ elements for some $k \leq n$ (here $2 k$ is an integer, but $k$ may be half-integral). Using a generator matrix $G$, such a code can be cast as the $\mathbb{F}_{2}$-span of its rows. To every linear $I$-code $C$ is attached an additive $\mathbb{F}_{4}$-code $\phi(C)$ by the alphabet substitution

$$
0 \rightarrow 0, a \rightarrow \omega, b \rightarrow 1, c \rightarrow \omega^{2},
$$

where $\mathbb{F}_{4}=\mathbb{F}_{2}[\omega]$, extended naturally to $\mathbb{F}_{4}^{n}$. Trivially, the Hamming weight of $\phi(x)$ is $w t(x)$.

We use the Magma notation

$$
\left[<0,1>, \cdots,<i, A_{i}>, \cdots,<n, A_{n}>\right]
$$

for the weight distribution of an additive $\mathbb{F}_{4}$-code code, where $A_{i}$ is the number of codewords of weight $i$. Two $I$-codes are permutation equivalent if there is a permutation of coordinates that maps one to the other (and, consequently, the $\phi$-image of one on the $\phi$-image of the other). The automorphism group $A u t(C)$ of an $I$-code $C$ is the group of column permutations that leave $C$ invariant.

\subsection{Duality}

Define an inner product on $I^{n}$, by writing $(x, y)=\sum_{i=1}^{n} x_{i} y_{i}$ for all $x, y \in I^{n}$.

The dual $C^{\perp}$ of a linear $I$-code $C$ is the module defined by

$$
C^{\perp}=\left\{y \in I^{n} \mid \forall x \in C,(x, y)=0\right\} .
$$

Thus the dual of a module is a module. A code is self-dual if it is equal to its dual.

Remark 1 The repetition code of length 2 is the linear $I$-code defined by $R_{2}:=\{00, a a, b b, c c\}$. Its dual is $R_{2}^{\perp}=\{00, a a, b b, c c, 0 b, b 0, a c, c a\}$, a supercode of $R_{2}$ of size 8 . In length one, we have $J^{\perp}=I$.

Remark 1 shows that the product of the sizes of a code and its dual is not always $4^{n}$. A code $C$ of length $n$ is nice if $|C|\left|C^{\perp}\right|=4^{n}$. A linear $I$-code $C$ is self-orthogonal if

$$
\forall x, y \in C,(x, y)=0 .
$$

Clearly, $C$ is self-orthogonal if and only if $C \subseteq C^{\perp}$.

A code of length $n$ is quasi self-dual (or QSD for short) if it is self-orthogonal and of size $2^{n}$. Note that the direct sum of two QSD codes is QSD. 
Following a terminology from [7], a quasi self-dual code over $I$ with all weights even is called a Type IV code. This notion is also stable by taking direct sums.

Remark 2 The repetition code of length 2 is quasi self-dual over $I$ and is of Type IV. This shows, by taking direct sums of codes, that Type IV codes over $I$ exist for all even lengths. We see that $J$ is a quasi self-dual code over $I$. This shows, again by taking direct sums, that QSD codes exist for all integer lengths.

We introduce the notion of Quasi Type IV code (QT4) as a QSD code with an even torsion code (see next section for a definition of torsion code). Every Type IV code is quasi Type IV but not conversely as the next example shows. The motivation will appear in the mass formula section.

Example 1 The code with three generators $\left(\begin{array}{llll}a & b & a & b \\ 0 & b & b & 0 \\ b & 0 & 0 & b\end{array}\right)$ is QSD but not Type IV as the sum of first and second row has odd weight. But its torsion code with generator matrix $\left(\begin{array}{llll}1 & 0 & 1 & 0 \\ 0 & 1 & 1 & 0 \\ 1 & 0 & 0 & 1\end{array}\right)$ is an even code.

\section{Structure of linear codes}

Let $C$ be a code of length $n$ over $I$. With this code we associate two binary codes of length $n$ :

1. the residue code defined by $\operatorname{res}(C)=\{\alpha(y) \mid y \in C\}$,

2. the torsion code defined by $\operatorname{tor}(C)=\left\{x \in \mathbb{F}_{2}^{n} \mid b x \in C\right\}$.

Recall that the Trace function of $\mathbb{F}_{4}$ down to $\mathbb{F}_{2}$ is defined by $\forall z \in \mathbb{F}_{4}, \operatorname{Tr}(z)=z+z^{2}$. In other words

$$
\operatorname{Tr}(0)=\operatorname{Tr}(1)=0 ; \operatorname{Tr}(\omega)=\operatorname{Tr}\left(\omega^{2}\right)=1 .
$$

By distinguishing four cases and induction on $n$ it can be checked that for all $x \in I^{n}$, we have $\operatorname{Tr}(\phi(x))=\alpha(x)$, and thus $\operatorname{res}(C)=\operatorname{Tr}(\phi(C))$. Similarly, we see that $\operatorname{tor}(C)$ is the so-called subfield subcode of $\phi(C)$, that is $\phi(C) \bigcap \mathbb{F}_{2}^{n}$. Denote by $\alpha_{C}$ the restriction of $\alpha$ to $C$. We see that $\operatorname{tor}(C) b=\operatorname{Ker}\left(\alpha_{C}\right)$, and that $\operatorname{res}(C)=\operatorname{Im}\left(\alpha_{C}\right)$. By the first isomorphism theorem applied to the map $\alpha_{C}$, we see that $|C|=|\operatorname{res}(C)||\operatorname{tor}(C)|$. There is a relationship between these two codes.

Lemma 1 If $C$ is a linear $I$-code then $\operatorname{res}(C) \subseteq \operatorname{tor}(C)$.

Proof Write an arbitrary codeword $z$ in $b$-adic decomposition form as $z=a x+b y$, with $x, y$ binary vectors. Since $\alpha(a x+b y)=x$, we have $x \in \operatorname{res}(C)$. Note that, by 
definition of the residue code, any $x \in \operatorname{res}(C)$ arises in that way. Multiplying the codeword $a x+b y$ by $a$, we see that $b x \in C$, implying $x \in \operatorname{tor}(C)$.

We let $k_{1}=\operatorname{dim}(\operatorname{res}(C))$, and $k_{2}=\operatorname{dim}(\operatorname{tor}(C))-k_{1}$, a nonegative quantity by Lemma 1 , and say that $C$ is of type $\left(k_{1}, k_{2}\right)$. It can be seen that $C$ is free as an $I$-module if and only if $k_{2}=0$. Further, by a previous observation, $|C|=|\operatorname{res}(C)||\operatorname{tor}(C)|=2^{2 k_{1}+k_{2}}$. We give a characterization of the generator matrix of a linear code as a function of these invariants.

Theorem 1 Assume $C$ is an $I$-linear code of length $n$ and type $\left(k_{1}, k_{2}\right)$. Then, up to a permutation of columns, there exists a generator matrix $G$ of $C$ that is of the form

$$
G=\left(\begin{array}{ccc}
a I_{k_{1}} & a X & Y \\
0 & b I_{k_{2}} & b Z
\end{array}\right)
$$

where $I_{j}$ denotes the identity matrix of order $j$, the matrix $Y$ has entries in $I$, and $X, Z$ are binary matrices.

Proof Write the generator matrices of $\operatorname{res}(C)$ and $\operatorname{tor}(C)$ as $G_{1}=\left(I_{k_{1}} X \alpha(Y)\right)$ and $G_{2}=\left(\begin{array}{ccc}I_{k_{1}} & X & \alpha(Y) \\ 0 & I_{k_{2}} & Z\end{array}\right)$, respectively, with $Y$ a matrix of suitable dimensions with entries in $I$, and $X, Z$ are binary matrices. By the first isomorphism theorem applied to the map $\alpha_{C}$, the matrix $G$ can be written on the form $\left(\begin{array}{c}R \\ b T\end{array}\right)$, where

$$
\operatorname{tor}(C)=\operatorname{res}(C) \oplus\langle T\rangle,
$$

and $\alpha(R)=G_{1}$. By taking linear combinations with rows of $T$, the matrix $R$ can be written in the above form.

Theorem 2 Assume $C$ is a nice I-linear code of length $n$ and type $\left(k_{1}, k_{2}\right)$. Then a parity check matrix $H$ of $C$ consistent with Theorem 1 is of the form

$$
H=\left(\begin{array}{lll}
Y^{t}+a Z^{t} X^{t} & a Z^{t} & a I_{n-k_{1}-k_{2}} \\
b X^{t} & b I_{k_{2}} & 0
\end{array}\right),
$$

where $I_{j}$ denotes the identity matrix of order $j$, and $X, Y, Z$ are as in Theorem 1. In particular $C^{\perp}$ is of type $\left(n-k_{1}-k_{2}, k_{2}\right)$. If, furthermore $C$ is self-dual, then $n=2 k_{1}+k_{2}$.

Proof A direct calculation shows that $H G^{t}=0$. This shows that, as $I$-modules, $\langle H\rangle=I^{n-k_{1}} H \subseteq C^{\perp}$. Equality follows by size comparison upon noticing that $\langle H\rangle$ has type $\left(n-k_{1}-k_{2}, k_{2}\right)$, and upon observing that by the niceness hypothesis we have $|C|\left|C^{\perp}\right|=4^{n}$. The last assertion follows by unicity of the type $k_{1}=n-k_{1}-k_{2}$.

To prepare for the derivation of a mass formula, we develop a method similar to that of [9]. We define a map $F$ from $\operatorname{res}(C)$ to $\mathbb{F}_{2}^{n} / \operatorname{tor}(C)$ by the formula 


$$
F(x)=\left\{y \in \mathbb{F}_{2}^{n} \mid a x+b y \in C\right\} .
$$

Since $C$ is $I$-linear, it follows that $F$ is $\mathbb{F}_{2}$-linear. We write $\psi(C)=(\operatorname{res}(C), \operatorname{tor}(C), F)$. The following Theorem is immediate, but essential.

Theorem 3 Keep the above notation. The map $\psi$ is a one to one correspondence between linear I-codes $C$ of length $n$, and triplets $\left(C_{1}, C_{2}, F\right)$ such that $C_{1}, C_{2}$ are binary codes of length $n$, satisfying $C_{1} \subseteq C_{2}$ and such that the map $F$ is $\mathbb{F}_{2}$-linear from $C_{1}$ to $\mathbb{F}_{2}^{n} / C_{2}$.

Proof Let $C$ be an arbitrary linear $I$-code. Let $\psi(C)=\left(C_{1}, C_{2}, F\right)$, be given. Writing an arbitrary codeword of $C$ as $a x+b y$, with $x, y$ binary vectors of length $n$, we see that

- $\alpha(a x+b y)=x$, implying $x \in C_{1}$;

- $y \in F(x)$, by definition of $F$.

From there it follows that the code $C$ is uniquely determined as

$$
C=\bigcup_{x \in C_{1}} \bigcup_{y \in F(x)} a x+b y \text {. }
$$

\section{Construction of QSD codes}

We call the next construction of I-codes from binary codes the multilevel construction.

Theorem 4 Let $C_{1}$ be a self-orthogonal binary code of length $n$, and let $C_{2}$ be a binary code of length $n$, where $C_{1} \subseteq C_{2}$. The code $C$ defined by the relation

$$
C=a C_{1}+b C_{2} \text {, }
$$

is a self-orthogonal code. Its residue code is $C_{1}$ and its torsion code is $C_{2}$. If, furthermore, $\left|C_{1}\right|\left|C_{2}\right|=2^{n}$, then $C$ is $Q S D$.

Proof The code $C$ is closed under addition, by linearity of $C_{1}$ and $C_{2}$. Let us check it is closed under multiplication by a scalar of $I$. Note that $a C=a^{2} C_{1}+a b C_{2}=b C_{1} \subseteq b C_{2} \subseteq C$. Likewise, we see that $c C=c a C_{1}+c b C_{2}=b C_{1} \in C$. Because $b I=\{0\}$, we get $b C=b a C_{1}+b^{2} C_{2}=\{0\} \subseteq C$.

Thus $C$ is $I$-linear.

For all $x, x^{\prime}$ in $C_{1}$ and $y, y^{\prime}$ in $C_{2}$ we have the inner products 


$$
\left(a x+b y, a x^{\prime}+b y^{\prime}\right)=a^{2}\left(x, x^{\prime}\right)+a b\left(x, y^{\prime}\right)+b a\left(y, x^{\prime}\right)+b^{2}\left(y, y^{\prime}\right)=b\left(x, x^{\prime}\right)=0
$$

since $x \in C_{1}$ which is self orthogonal. Thus $C$ is self-orthogonal. The residue and torsion codes are immediately derived from the definitions. Since $|C|=\left|C_{1}\right|\left|C_{2}\right|$, the last assertion follows.

Note that for given residue and torsion codes many QSD codes may exist. As an example, the code $C_{1}$ generated by the matrix $\left(\begin{array}{ccc}a & a & 0 \\ 0 & b & b\end{array}\right)$ and the code $C_{2}$ generated by the matrix $\left(\begin{array}{ccc}a & a & b \\ 0 & b & b\end{array}\right)$ have same residue and torsion codes.

\section{Mass formulas}

\subsection{QSD codes}

Define $N_{I}(n, k)$ as the number of QSD $I$-codes of length $n$ and type $(k, n-2 k)$. Let $\Phi(N, K)$ denote the number of self-orthogonal binary codes having parameters $[N, K]$. The following explicit formula for this quantity can be found in $[10, \mathrm{p} .1]$. It is valid for $K \geq 1$.

$$
\Phi(N, K)= \begin{cases}\frac{\prod_{j=1}^{K}\left(2^{N+1-2 j}-1\right)}{\prod_{j=1}^{K}\left(2^{j}-1\right)} & \text { if } \mathrm{n} \geq 3 \text { is odd }, \\ \frac{\left(2^{N-K}-1\right) \prod_{j=1}^{K-1}\left(2^{N-2 j}-1\right)}{\prod_{j=1}^{K}\left(2^{j}-1\right)} & \text { if } \mathrm{n} \geq 2 \text { is even } .\end{cases}
$$

We need the following counting lemma. For information on $q$-binomial coefficients we refer the reader to [11, p. 443].

Lemma 2 The number $E(q, n, r, s)$ of subspaces of dimension $r$ of $\mathbb{F}_{q}^{n}$ containing a given subspace of dimension $s<r$ is $\left(\begin{array}{c}n-s \\ r-s\end{array}\right)_{q}$, where $\left(\begin{array}{l}i \\ i\end{array}\right)_{q}$ denotes the $q$-binomial coefficient defined for integers $i \geq j$ by

$$
\left(\begin{array}{l}
i \\
j
\end{array}\right)_{q}=\frac{\left(q^{i}-1\right)\left(q^{i-1}-1\right) \cdots\left(q^{i-j+1}-1\right)}{\left(q^{j}-1\right)\left(q^{j-1}-1\right) \cdots(q-1)} .
$$

Proof Follows by Lemma 3 of [6] with $i=j=s$ and $k=r$.

We also need the following simple Lemma.

Lemma 3 If $C$ is a self-orthogonal I-code, then its residue res $(C)$ is a binary selforthogonal code.

Proof By linearity of the map $\alpha$ it can be seen that $\forall x, y \in C$, we have 


$$
(\alpha(x), \alpha(y))=\alpha((x, y))=\alpha(0)=0 .
$$

The result follows by $\operatorname{res}(C)=\operatorname{Im}\left(\alpha_{C}\right)$.

We can now count the QSD codes of given length and residue dimension.

Theorem 5 For all lengths $n$ and type $(k, n-2 k)$, with $k>0$ we have

$$
N_{I}(n, k)=\Phi(n, k)\left(\begin{array}{c}
n-k \\
n-2 k
\end{array}\right)_{2} 2^{k^{2}} .
$$

Proof Let $C$ be a QSD code of length $n$ and type $(k, n-2 k)$. We reason in terms of the map $\psi$. Note the following three independent counts.

1. By Lemma 3, we know that $\operatorname{res}(C)$ is self-orthogonal. By definition of $\Phi$, there are $\Phi(n, k)$ self-orthogonal binary $[n, k]$ codes that can be used as $\operatorname{res}(C)$.

2. The residue code is contained in $E(2, n, n-k, k)=\left(\begin{array}{c}n-k \\ n-2 k\end{array}\right){ }_{2}$ possible torsion codes by Lemma 2 for $r=k, s=n-k$, and $q=2$.

3. The additive map $F$ is arbitrary from $\operatorname{res}(C)$ to $\mathbb{F}_{2}^{n} / \operatorname{tor}(C)$. By the QSD hypothesis, these two vector spaces have dimension $k$. Hence, by considering the matrix of $F$, we see that there are $2^{k^{2}}$ choices for $F$.

The result follows by multiplying the three factors together.

The following mass formula follows by the usual counting technique.

Corollary 1 For given length $n$ and type $(k, n-2 k)$, with $k>0$, we have

$$
\sum_{C} \frac{1}{|\operatorname{Aut}(C)|}=\frac{\Phi(n, k)\left(\begin{array}{c}
n-k \\
n-2 k
\end{array}\right) 2^{k^{2}}}{n !},
$$

where $C$ runs over distinct representatives of equivalence classes under column permutations of QSD codes of length $n$ and type $(k, n-2 k)$.

\subsection{Quasi type IV codes}

Define $N_{4}(n, k)$ as the number of QT4 I-codes of length $n$ and type $(k, n-2 k)$. The analogue of Lemma 2 is as follows.

Lemma 4 The number $E E(n, r, s)$ of even codes of dimension $r$ of $\mathbb{F}_{2}^{n}$ containing a given subspace of dimension $s<r$ is $\left(\begin{array}{c}n-1-s \\ r-s\end{array}\right)_{2}$.

Proof Follows by Lemma 3 of [6] with $i=j=s$ and $k=r$ for $q=2$. Here $C$ in the notation of [6] is the binary code of length $n$, and dimension $n-1$, consisting of all even Hamming weight vectors. 
Using this Lemma the following analogue of Theorem 5 for QT4 codes can be shown. The easy proof is omitted.

Theorem 6 For all lengths $n$ and type $(k, n-2 k)$, with $k>0$ we have

$$
N_{4}(n, k)=\Phi(n, k)\left(\begin{array}{c}
n-1-k \\
n-2 k
\end{array}\right) 2_{2}^{k^{2}} .
$$

The following mass formula follows by the usual counting technique.

Corollary 2 For given length $n$, and type $(k, n-2 k)$, with $k>0$, we have

$$
\sum_{C} \frac{1}{|A u t(C)|}=\frac{\Phi(n, k)\left(\begin{array}{c}
n-1-k \\
n-2 k
\end{array}\right) 2^{k^{2}}}{n !}
$$

where $C$ runs over distinct representatives of equivalence classes under column permutations of QT4 codes of length $n$ and type $(k, n-2 k)$.

\section{Short length classification $(n<4)$}

In the following, we classify, up to coordinate permutation, QSD codes. We construct QSD codes by means of the multilevel construction, and of the characterization of Theorem 1 , in short lengths $(n<4)$ and rely on the mass formula to know the maximum number of equivalence classes.

For $n=1$ there is just one QSD code with generator matrix $(b)$.

\section{$6.1 n=2$}

There are two codes of length 2. Both are Type IV codes and their weight distribution is $[<0,1>,<2,3>]$. One is generated by $(a a)$ and the other one by $(a c)$.

\section{$6.2 n=3$}

There are exactly six codes that are not permutation equivalent. All these codes have an automorphism group of order 2 . The following four codes have a minimum distance equal to 1 .

- One code has generator matrix

$$
\left(\begin{array}{lll}
a & a & b \\
0 & b & 0
\end{array}\right)
$$

and weight distribution $[<0,1>,<1,2>,<2,1>,<3,4>]$.

- One code has generator matrix 


$$
\left(\begin{array}{lll}
a & a & 0 \\
0 & b & 0
\end{array}\right)
$$

and weight distribution $[<0,1>,<1,2>,<2,5>]$.

- The last two codes amongst these four share the same weight distribution $[<0,1>,<1,1>,<2,3>,<3,3>]$. Their generator matrices are

$$
\left(\begin{array}{lll}
a & 0 & a \\
0 & b & 0
\end{array}\right)
$$

and

$$
\left(\begin{array}{lll}
a & 0 & c \\
0 & b & 0
\end{array}\right)
$$

Two codes have minimum distance 2 and weight distribution $[<0,1>,<2,5>,<3,2>]$. Their generator matrices are

$$
\left(\begin{array}{lll}
a & a & b \\
0 & b & b
\end{array}\right)
$$

and

$$
\left(\begin{array}{lll}
a & a & 0 \\
0 & b & b
\end{array}\right) .
$$

These two codes are quasi Type IV codes with, as torsion code, a binary code of parameters $[3,2,2]$ and generator matrix $\left(\begin{array}{lll}b & 0 & b \\ 0 & b & b\end{array}\right)$. Note that we obtain two quasi Type IV codes, which is consistent with Corollary 1.

\section{Conclusion}

In this article, we have studied quasi self-dual codes over the non-unital commutative ring $I$ of order four. The existence of codes that are not nice precludes any attempt to derive a general MacWilliams formula, since this would imply a relation between the size of a code and that of its dual. We have thus introduced QSD codes as an alternative to the concept of self-dual codes. For similar reasons, we have introduced the notion of quasi Type IV codes, in view of the difficulty of studying Type IV codes. We have derived a mass formula for QSD codes and for QT4 codes to classify them under coordinate permutation equivalence.

The main open problem arising from this work would be to derive a mass formula for Type IV codes. This would require a characterization of these codes akin to what exists over $E$ [1], or over unital rings of Type IV [7]. It would be interesting to derive a classification under a notion of equivalence based on the symmetry of the 
alphabet between $a$ and $c$. It might be a simpler classification than the classification under column permutation of the present paper.

On the computational side, pushing the classification of QSD codes beyond length 3 , is certainly a worthy project, and might lead to the discovery of new additive $\mathbb{F}_{4}$-codes in lengths $>3$.

Acknowledgements This project was funded by the Deanship of Scientific Research (DSR) at King Abdulaziz University, Jeddah, under grant number KEP-PhD-29-130-40. The authors, therefore, acknowledge with thanks DSR for technical and financial support.

\section{References}

1. Alahmadi, A., Bonnecaze, A., Shoaib, H., Altassan, A., Bassafar, W., Solé, P.: Type IV codes over a non-unital ring, submitted

2. Balmaceda, J.M.L., Betty, R.A.L., Nemenzo, F.: Mass formula for self-dual codes over $\mathbb{Z}_{p^{2}}$. Discrete Math. 308, 2984-3002 (2008)

3. Calderbank, A.R., Rains, E.M., Sloane, N.J.A.: Quantum error correction via codes over $G F(4)$. IEEE Trans. Inf. Theory 44, 1369-1387 (1998)

4. Conway, J.H., Sloane, N.J.A.: Self-dual codes over the integers modulo four. J. Comb. Theory Ser. A 62, 30-45 (1993)

5. Choi, W.: Mass formula of self-dual codes over Galois rings $G R\left(p^{2}, 2\right)$. Korean J. Math. 24(4), 751764 (2016)

6. Delsarte, P.: Association schemes and t-designs in regular semi-lattices. J. Comb. Theory 20, 230243 (1976)

7. Dougherty, S.T., Gaborit, P., Harada, M., Munemasa, A., Solé, P.: Type IV self-dual codes over rings. IEEE Trans. Inf. Theory 45(7), 2345-2360 (1999)

8. Fine, B.: Classification of finite rings of order $p^{2}$. Math. Mag. 66(4), 248-252 (1993)

9. Gaborit, P.: Mass formulas for self-dual codes over $\mathbb{Z}_{4}$ and $\mathbb{F}_{q}+u_{\mathbb{F}} q$ rings. IEEE Trans. Inf. Theory 42, 1222-1228 (1996)

10. Hou, X.D.: On the number of inequivalent binary self-orthogonal codes. Trans. Inf. Theory 53, 2459-2479 (2007)

11. MacWilliams, F.J., Sloane, N.J.A.: The Theory of Error-Correcting Codes. North-Holland, Amsterdam (1977)

12. http://magma.maths.usyd.edu.au/magma/

13. Raghavendran, R.: A class of finite rings. Compos. Math. 21, 195-229 (1969)

14. Shi, M., Alahmadi, A., Solé, P.: Codes and Rings: Theory and Practice. Academic Press, Cambridge (2017)

15. Wood, Jay A.: Duality for modules over finite rings and applications to coding theory. Am. J. Math. 121(3), 555-575 (1999)

Publisher's Note Springer Nature remains neutral with regard to jurisdictional claims in published maps and institutional affiliations. 


\section{Terms and Conditions}

Springer Nature journal content, brought to you courtesy of Springer Nature Customer Service Center GmbH ("Springer Nature").

Springer Nature supports a reasonable amount of sharing of research papers by authors, subscribers and authorised users ("Users"), for small-scale personal, non-commercial use provided that all copyright, trade and service marks and other proprietary notices are maintained. By accessing, sharing, receiving or otherwise using the Springer Nature journal content you agree to these terms of use ("Terms"). For these purposes, Springer Nature considers academic use (by researchers and students) to be non-commercial.

These Terms are supplementary and will apply in addition to any applicable website terms and conditions, a relevant site licence or a personal subscription. These Terms will prevail over any conflict or ambiguity with regards to the relevant terms, a site licence or a personal subscription (to the extent of the conflict or ambiguity only). For Creative Commons-licensed articles, the terms of the Creative Commons license used will apply.

We collect and use personal data to provide access to the Springer Nature journal content. We may also use these personal data internally within ResearchGate and Springer Nature and as agreed share it, in an anonymised way, for purposes of tracking, analysis and reporting. We will not otherwise disclose your personal data outside the ResearchGate or the Springer Nature group of companies unless we have your permission as detailed in the Privacy Policy.

While Users may use the Springer Nature journal content for small scale, personal non-commercial use, it is important to note that Users may not:

1. use such content for the purpose of providing other users with access on a regular or large scale basis or as a means to circumvent access control;

2. use such content where to do so would be considered a criminal or statutory offence in any jurisdiction, or gives rise to civil liability, or is otherwise unlawful;

3. falsely or misleadingly imply or suggest endorsement, approval, sponsorship, or association unless explicitly agreed to by Springer Nature in writing;

4. use bots or other automated methods to access the content or redirect messages

5. override any security feature or exclusionary protocol; or

6. share the content in order to create substitute for Springer Nature products or services or a systematic database of Springer Nature journal content.

In line with the restriction against commercial use, Springer Nature does not permit the creation of a product or service that creates revenue, royalties, rent or income from our content or its inclusion as part of a paid for service or for other commercial gain. Springer Nature journal content cannot be used for inter-library loans and librarians may not upload Springer Nature journal content on a large scale into their, or any other, institutional repository.

These terms of use are reviewed regularly and may be amended at any time. Springer Nature is not obligated to publish any information or content on this website and may remove it or features or functionality at our sole discretion, at any time with or without notice. Springer Nature may revoke this licence to you at any time and remove access to any copies of the Springer Nature journal content which have been saved.

To the fullest extent permitted by law, Springer Nature makes no warranties, representations or guarantees to Users, either express or implied with respect to the Springer nature journal content and all parties disclaim and waive any implied warranties or warranties imposed by law, including merchantability or fitness for any particular purpose.

Please note that these rights do not automatically extend to content, data or other material published by Springer Nature that may be licensed from third parties.

If you would like to use or distribute our Springer Nature journal content to a wider audience or on a regular basis or in any other manner not expressly permitted by these Terms, please contact Springer Nature at 